\title{
A Multivariate Innovations State Space Beveridge-Nelson Decomposition
}

\author{
Ashton de Silva ${ }^{a *}$, Rob J Hyndman ${ }^{b}$ and Ralph Snyder $^{b}$ \\ ${ }^{a}$ School of Economics, Finance and Marketing, RMIT University, VIC 3000, Australia. \\ ${ }^{b}$ Department of Econometrics and Business Statistics, Monash University, VIC 3800, Australia.
}

Revised-Dec 2008

\begin{abstract}
The Beveridge-Nelson vector innovations structural time series framework is a new formulation that decomposes a set of variables into their permanent and transitory components. The proposed framework is flexible, modelling inter-series relationships and common features in a simple manner. In particular, it is shown that this new specification is simpler than conventional state space and cointegration approaches. The approach is illustrated using a trivariate data set comprising the GDP of Australia, the USA and the UK.
\end{abstract}

Keywords: vector innovations structural time series model, multivariate time series model, Beveridge-Nelson decomposition, common components.

\section{Introduction}

Decomposing variables into permanent and transitory components has been an important theme of economic research for more than 25 years. A new multivariate framework that disaggregates a collection of variables into permanent and transitory components is presented in this paper. 
Specifically, the vector innovations structural time series (hereafter VISTS, de Silva et al. 2007) framework is modified to extract permanent and transitory components in accordance with the Beveridge-Nelson (hereafter BN, Beveridge \& Nelson 1981) decomposition. These models will be denoted as BN-VISTS. The specification presented is a multivariate extension for Anderson, Low \& Snyder (2004, 2006) and Hyndman et al. (2008, chapter 20).

An important advantage of this multivariate approach is that common permanent or transitory components can be evaluated using standard diagnostic tools, e.g., information criteria. This is different from Stock \& Watson (1988a) and Vahid \& Engle (1993) who developed statistical testing procedures based on the cointegration specification. Arguably, the proposed approach is easier.

The structure of the paper is as follows: a brief history of time series decompositions are summarised in the next section; the new framework, its models and implementation are outlined in section 3; the new specification applied to real GDP of Australia, the United Kingdom and the United States is presented in section 4; and finally, the findings are summarised in section 5 .

\section{Background}

Traditionally, analysis of time series involves disaggregation of the observations into a set of interpretable components. One such decomposition is:

$$
Y_{t}=T_{t}+C_{t}+S_{t}+I_{t}
$$

where $T_{t}, C_{t}, S_{t}$ and $I_{t}$ conventionally denote the trend, cyclical, seasonal and irregular component at time $t$. A more recent decomposition is that by Beveridge \& Nelson (1981) who proposed that a macroeconomic variable can be disaggregated into a stationary and non-stationary component. These components are denoted as the transitory and permanent components respectively.

Beveridge \& Nelson (1981) tailor the permanent/transitory decomposition to the stochastic properties of the data. The permanent component almost always comprises 
a random walk with the same rate of drift as in the original data. In addition, the permanent component also comprises a disturbance term proportional to that of the original data. The transitory component represents the "forecastable momentum present at each time period" (Beveridge \& Nelson 1981, page 158) and is expected to dissipate as the series tends to its permanent level.

In general, two different formulations have been used to measure the trend (permanent) and transitory ${ }^{1}$ (temporary) components. One is the ARIMA specification which is advocated by Beveridge \& Nelson (1981); the other is the structural time series approach advocated by Harvey \& Jaeger (1993) and others.

The results from these two procedures are typically very different. The ARIMA specification commonly yields a dominant permanent component whereas the structural time series approach often yields a dominant transitory component. In recent times there has been an attempt to reconcile these two approaches (see Morley, Nelson \& Zivot 2003, Proietti 2002). These papers compare both the analytical and empirical aspects of the two specifications.

Analytically, the conclusion reached is that any structural time series model has an ARIMA representation, but not vice-versa. This is directly attributable to the form of the structural time series model adopted. Specifically, the random disturbances of the permanent and temporary components are constrained to be independent of each other (This issue was discussed in Morley et al. 2003).

Using US GNP, Morley et al. (2003) find no evidence to suggest that the estimated correlation between the permanent and temporary components is zero. Moreover, the estimates seem to suggest an almost perfect negative correlation between the two components. A similar outcome is observed for Italian real GDP (Proietti 2002).

This finding endorses the formulation proposed by Anderson et al. (2006), which assumes the disturbances of the latent components are perfectly correlated. Specifically, Anderson et al. (2006) present an innovations structural time series model (Hyndman et al. 2002). In this paper, we extend this formulation to a multivariate con-

\footnotetext{
${ }^{1}$ Beveridge \& Nelson (1981) and others (including Harvey \& Jaeger 1993), use the term "cycle" to denote the transitory component. We believe the use of this term can cause unnecessary confusion, as the literature appears to be unclear on the precise definition of a cycle (Pagan 1997), hence we refer to this component as transitory.
} 
text.

There are two streams of research on multivariate $\mathrm{BN}$ decompositions: the first relating to traditional state space specifications; the second focusing on modelling and testing for common permanent and transitory components within a cointegrating framework.

Two papers that have considered the multivariate BN decomposition in the form of traditional state space models are Morley (2002) and Sinclair (2005). Sinclair (2005) provides a detailed analysis of the American unemployment rate and GDP paying particular attention to Okun's law. Morley (2002) outlines an analytical form only.

The main contributions to the second stream are King, Plosser, Stock \& Watson (1987), Stock \& Watson (1988a), Vahid \& Engle (1993, 1997) and Barillas \& Schleicher (2003). These papers consider both VAR and traditional time series specifications. A particular emphasis in two of these articles (Stock \& Watson 1988a, Vahid \& Engle 1993) is the ability to formally test for the presence of common features. In particular, Stock \& Watson (1988a) formulate a test to determine common-trends based on autoregressive matrices. Similarly, Vahid \& Engle (1993) derive a common-cycle test for series that are cointegrated.

In addition to formulating a common-cycle test Vahid \& Engle (1993) review what they define as the Beveridge-Nelson-Stock-Watson (BNSW) decomposition. This decomposition relates the cointegration specification to a structural time series framework. A brief review of the derivation is presented here.

The derivation begins by stating the Wold decomposition for a vector of $N$ at time $t$ $\left(\boldsymbol{y}_{t}\right)$, integrated of order one

$$
\Delta \boldsymbol{y}_{t}=\boldsymbol{\mu}+C(L) \boldsymbol{\epsilon}_{t},
$$

where $C(L)=\sum_{j=1}^{\infty} C_{j} L^{j}$, a matrix polynomial function of the lag operator $L$ where $L \boldsymbol{y}_{t}=\boldsymbol{y}_{t-1}, \Delta=1-L$ and $C_{0}=I_{N}$. For simplicity, equation (2.1) can be rewritten as

$$
\Delta \boldsymbol{y}_{t}=\boldsymbol{\mu}+C(1) \varepsilon_{t}+\Delta C^{*}(L) \varepsilon_{t}
$$


where $C_{i}^{*}=-\sum_{j>i}^{\infty} C_{j}$ for all $i$ and $C_{0}^{*}=I_{N}-C(1)$. Integrating both sides of (2.2) gives

$$
\boldsymbol{y}_{t}=\left(\boldsymbol{\mu}+C(1) \varepsilon_{t-s}\right) /(1-L)+C^{*}(L) \varepsilon_{t}
$$

Equation (2.3) denotes the multivariate $\mathrm{BN}$ decomposition, where the first term depicts the permanent (trend) component and the second, the transitory (temporary) component. If a common trend exists (that is, if a cointegrating relationship between two or more variables exist) then $C(1)$ will be of reduced rank. Specifically, the rank of $C(1)$ will equal $k$, where $k<N$ and $C(1)$ can be expressed in the form:

$$
C(1)=\gamma \boldsymbol{\delta}^{\prime}
$$

where $\boldsymbol{\gamma}$ and $\boldsymbol{\delta}$ are both of rank $k$. Cointegration also implies that there exists a $k$-vector $\boldsymbol{b}$ of common drifts such that $\boldsymbol{\mu}=\boldsymbol{\gamma} \boldsymbol{b}$. By denoting the trend and temporary components at time $t$ to be $\boldsymbol{\tau}_{t}$ and $\boldsymbol{c}_{t}$ respectively (where $\boldsymbol{\tau}_{t}$ is a vector of length $k$ and $\boldsymbol{c}_{t}$ a vector of length $N$ ), the common trend BNSW decomposition is expressed as

$$
\boldsymbol{y}_{t}=\gamma \boldsymbol{\tau}_{t}+\boldsymbol{c}_{t}
$$

where

$$
\boldsymbol{\tau}_{t}=\boldsymbol{b}+\boldsymbol{\tau}_{t-1}+\boldsymbol{\delta}^{\prime} \varepsilon_{t}
$$

Equation (2.4) is the common trend specification of the BN decomposition. Watson (1994) refers to this representation as the common trends representation of the cointegrated system. In section 3.3 it is shown how the new multivariate formulation relates to the BNSW form defined by (2.3).

\section{Models}

In this section the general form of the VISTS framework is presented. Three special cases are also discussed which form the basis of the empirical work presented in Section 4. 


\subsection{VISTS general form}

The VISTS general form is

$$
\begin{aligned}
\boldsymbol{y}_{t} & =H \boldsymbol{x}_{t-1}+\boldsymbol{e}_{t} \\
\boldsymbol{x}_{t} & =F \boldsymbol{x}_{t-1}+A \boldsymbol{e}_{t} \\
\boldsymbol{e}_{t} & \stackrel{i i d}{\sim} \operatorname{MVN}(\mathbf{0}, \Sigma),
\end{aligned}
$$

where $H, F$ and $A$ denote various coefficient matrices, $\boldsymbol{x}_{t}$ denotes a $k$-vector of states and $\boldsymbol{e}_{t}$ denotes an $N$-vector of innovations which are normally distributed at time $t$. It is assumed that $\Sigma$, the covariance matrix of $\boldsymbol{e}_{t}$, is diagonal.

Equations (3.1) and (3.2) denote the measurement and transition equation respectively. The measurement equation describes the vector of observations as a function of unobserved components. Each variable has a latent permanent and temporary component and is combined according to the structure matrix $H$.

The transition equation, a first order recursive process, describes how the components vary over time. This equation is a function of $F$, the transition matrix, the previous set of state values denoted by $\boldsymbol{x}_{t-1}$, and the permanent effect matrix $A$ which moderates the influence of unexpected changes.

The coefficient matrices $H$ and $F$ are typically partially predetermined. The permanent effect matrix, $A$, is wholly determined by the data. The dimension of these matrices will vary according to the model estimated.

It is important here to reiterate the difference between the VISTS specification and the conventional specification (Harvey \& Jaeger 1993). The VISTS framework has one source of error per series; conversely, the conventional specification has multiple sources of error per series and takes on the form

$$
\begin{aligned}
& \boldsymbol{y}_{t}=H \boldsymbol{x}_{t-1}+\boldsymbol{\eta}_{t}, \boldsymbol{\eta}_{t} \stackrel{i i d}{\sim} \operatorname{MVN}(\mathbf{0}, Q) \\
& \boldsymbol{x}_{t}=F \boldsymbol{x}_{t-1}+\varepsilon_{t}, \quad \varepsilon_{t} \stackrel{i i d}{\sim} \operatorname{MVN}(\mathbf{0}, R),
\end{aligned}
$$

where $Q$ and $R$ denote the covariance matrices of the measurement and transitory equa- 
tion respectively. The conventional structural time series multivariate model, denoted by (3.4), assumes a different source of error for the measurement and transition equations. Furthermore, it is most commonly assumed that $\mathrm{E}\left(\boldsymbol{\eta}_{t}, \boldsymbol{\varepsilon}_{s}\right)=0$ for $t, s=1, \ldots, T$. This difference, although appearing somewhat trivial, fundamentally differentiates these specifications. The multiple source of error specification is arguably more difficult to implement. In addition it can be shown that the conventional structural time series specification is a special case of VISTS framework (de Silva et al. 2007).

\subsection{Beveridge-Nelson Vector Innovations Structural Time Series models}

Anderson et al. (2004) consider three univariate innovations structural times series models. These models will be referred to as the BN-univariate innovations structural time series (BN-UISTS) models. The first was the BN-UISTS $(0,1,1)$ model which is equivalent to an $\operatorname{ARIMA}(0,1,1)$ process. It is specified by equations $(3.5)$ and $(3.6)$ :

$$
\begin{aligned}
y_{t} & =\mu+\left[\begin{array}{ll}
1 & 1
\end{array}\right]\left[\begin{array}{l}
m_{t-1} \\
c_{t-1}
\end{array}\right]+e_{t} \\
{\left[\begin{array}{l}
m_{t} \\
c_{t}
\end{array}\right] } & =\left[\begin{array}{l}
\mu \\
0
\end{array}\right]+\left[\begin{array}{ll}
1 & 0 \\
0 & 0
\end{array}\right]\left[\begin{array}{c}
m_{t-1} \\
c_{t-1}
\end{array}\right]+\left[\begin{array}{c}
\alpha \\
1-\alpha
\end{array}\right] e_{t},
\end{aligned}
$$

where the trend and transitory components at time $t$ is denoted by $m_{t}$ and $c_{t}$ respectively. The term $\mu$ denotes a drift term and the permanent effect parameter $\alpha$ measures the impact of unexpected changes on the trend. The response in the transitory part of the series is measured by $1-\alpha$.

An important characteristic of this specification is the adding-up constraint imposed on the coefficients of the error term in the state equations. Imposing this constraint means that when the state equations are substituted into the observation equation ${ }^{2}$, the only terms remaining are $m_{t}, c_{t}$ and $e_{t}$.

\footnotetext{
${ }^{2}$ Prior to the substitution, each equation is expressed in terms of its lagged term.
} 
The vector form of the model is labeled the BN-VISTS $(0,1,1)$ model. It has the form

$$
\begin{gathered}
\boldsymbol{y}_{t}=\boldsymbol{\mu}+\left[\begin{array}{ll}
I & I
\end{array}\right]\left[\begin{array}{l}
\boldsymbol{m}_{t-1} \\
\boldsymbol{c}_{t-1}
\end{array}\right]+\boldsymbol{e}_{t} \\
{\left[\begin{array}{l}
\boldsymbol{m}_{t} \\
\boldsymbol{c}_{t}
\end{array}\right]=\left[\begin{array}{l}
\boldsymbol{\mu} \\
\mathbf{0}
\end{array}\right]+\left[\begin{array}{ll}
I & O \\
O & O
\end{array}\right]\left[\begin{array}{l}
\boldsymbol{m}_{t-1} \\
\boldsymbol{c}_{t-1}
\end{array}\right]+\left[\begin{array}{c}
A \\
I-A
\end{array}\right] \boldsymbol{e}_{t},}
\end{gathered}
$$

where $I$ denotes an $N \times N$ identity matrix and the permanent component matrix $A$ is of dimension $N \times N$. The $O$ matrices denote $N \times N$ null matrices and $\mathbf{0}$ denote null vectors of length $N$. The term $\boldsymbol{\mu}$ denotes an $N$-vector of constants acting as a drift term. Bolded characters denote vectors of length $N$.

The next model Anderson et al. (2004) specified was the BN-UISTS(1,1,0). The purpose of this model was to to see how the persistence in the series could be managed without a MA term.

$$
\begin{gathered}
y_{t}=\mu+\left[\begin{array}{ll}
1 & -\phi
\end{array}\right]\left[\begin{array}{l}
m_{t-1} \\
c_{t-1}
\end{array}\right]+e_{t} \\
{\left[\begin{array}{l}
m_{t} \\
c_{t}
\end{array}\right]=\left[\begin{array}{l}
\mu \\
0
\end{array}\right]+\left[\begin{array}{cc}
1 & 0 \\
0 & -\phi
\end{array}\right]\left[\begin{array}{c}
m_{t-1} \\
c_{t-1}
\end{array}\right]+\left[\begin{array}{c}
\alpha \\
1-\alpha
\end{array}\right] e_{t} .}
\end{gathered}
$$

The reduced form of this model is an $\operatorname{ARIMA}(1,1,1)$. Accordingly, the following restriction is imposed to eliminate the MA term:

$$
\phi=\frac{1-\alpha}{\alpha}
$$

Therefore, although this model introduces a new parameter, it does not increase the number of unknowns because of the condition specified in (3.7). The multivariate form of 
the model denoted as the BN-VISTS $(1,1,0)$ is

$$
\begin{aligned}
\boldsymbol{y}_{t} & =\boldsymbol{\mu}+\left[\begin{array}{ll}
I & -\Phi
\end{array}\right]\left[\begin{array}{l}
\boldsymbol{m}_{t-1} \\
\boldsymbol{c}_{t-1}
\end{array}\right]+e_{t} \\
{\left[\begin{array}{l}
\boldsymbol{m}_{t} \\
\boldsymbol{c}_{t}
\end{array}\right] } & =\left[\begin{array}{l}
\boldsymbol{\mu} \\
\mathbf{0}
\end{array}\right]+\left[\begin{array}{cc}
I & O \\
O & -\Phi
\end{array}\right]\left[\begin{array}{c}
\boldsymbol{m}_{t-1} \\
\boldsymbol{c}_{t-1}
\end{array}\right]+\left[\begin{array}{c}
A \\
I-A
\end{array}\right] \boldsymbol{e}_{t} .
\end{aligned}
$$

As before, the autoregressive part of the model must be constrained to eliminate the MA term. The multivariate analogue of (3.7) being

$$
\Phi=(I-A) A^{-1}
$$

The final model that Anderson et al. (2004) proposed was the BN-UISTS $(2,1,2)$ specification:

$$
\begin{gathered}
y_{t}=\mu+\left[\begin{array}{lll}
1 & -\phi_{1} & 1
\end{array}\right]\left[\begin{array}{l}
m_{t-1} \\
c_{t-1} \\
d_{t-1}
\end{array}\right]+e_{t} \\
{\left[\begin{array}{l}
m_{t} \\
c_{t} \\
d_{t}
\end{array}\right]=\left[\begin{array}{l}
\mu \\
0 \\
0
\end{array}\right]+\left[\begin{array}{ccc}
1 & 0 & 0 \\
0 & -\phi_{1} & 1 \\
0 & -\phi_{2} & 0
\end{array}\right]\left[\begin{array}{c}
m_{t-1} \\
c_{t-1} \\
d_{t-1}
\end{array}\right]+\left[\begin{array}{c}
\alpha \\
1-\alpha \\
\theta_{1}
\end{array}\right] e_{t} .}
\end{gathered}
$$

The multivariate form of this model, called the $\operatorname{BN}-\operatorname{VISTS}(2,1,2)$ is

$$
\begin{gathered}
\boldsymbol{y}_{t}=\boldsymbol{\mu}+\left[\begin{array}{lll}
I & -\Phi_{1} & I
\end{array}\right]\left[\begin{array}{l}
\boldsymbol{m}_{t-1} \\
\boldsymbol{c}_{t-1} \\
\boldsymbol{d}_{t-1}
\end{array}\right]+\boldsymbol{e}_{t} \\
{\left[\begin{array}{l}
\boldsymbol{m}_{t} \\
\boldsymbol{c}_{t} \\
\boldsymbol{d}_{t}
\end{array}\right]=\left[\begin{array}{l}
\boldsymbol{\mu} \\
\mathbf{0} \\
\mathbf{0}
\end{array}\right]+\left[\begin{array}{ccc}
I & O & O \\
O & -\Phi_{1} & I \\
O & -\Phi_{2} & O
\end{array}\right]\left[\begin{array}{c}
\boldsymbol{m}_{t-1} \\
\boldsymbol{c}_{t-1} \\
\boldsymbol{d}_{t-1}
\end{array}\right]+\left[\begin{array}{c}
A \\
I-A \\
\Theta_{1}
\end{array}\right] \boldsymbol{e}_{t} .}
\end{gathered}
$$

This model is relatively large, requiring four parameter matrices of dimension $N \times N$ to be estimated. The matrices are denoted as $\Phi_{1}, \Phi_{2}, \Theta_{1}$ and $A$. 


\subsection{Identification and common component models}

In this section, two aspects of the BN-VISTS specification are considered. The first is the potential problem of identification, and the second is the common component specification.

The problem of identification when fitting VARMA models is well known (see Tiao \& Tsay 1989). Specifically, some autoregressive or moving average coefficients may be unidentifiable. For example in the case of an ARMA model the AR and MA coefficients will be unidentifiable if they have common factors. For a complete description of the identification problem with respect to vector ARMA models, and the various solutions available, refer to Athanasopoulos (2005).

The BN-VISTS $(2,1,2)$ can exhibit the same problem; that is, the AR and MA coefficients may not be uniquely identifiable. To negate this problem the $\Phi$ and $\Theta_{1}$ matrices are constrained to be diagonal. This is a simple and effective solution to the problem of identification (Dufour \& Pelletier 2005). It is important to note that no constraint is placed on $A$.

The second issue addressed in this section is the common component specification. A common component in the trend occurs when the rank of $A$ is less than $N$, therefore

$$
A=\gamma \boldsymbol{\delta}^{\prime}
$$

where $\boldsymbol{\gamma}$ and $\boldsymbol{\delta}$ correspond to matrices of rank $k$, such that $k<N$. For $A$ to be uniquely identifiable, either $\boldsymbol{\gamma}$ or $\boldsymbol{\delta}$ must be fixed. In the application that follows, the values of $\boldsymbol{\delta}$ are chosen according to economic theory (Johansen 1995).

When $A$ is of reduced rank the BN-VISTS specification corresponds to the common trends representation of a cointegrated system. The link between these two specifications is straightforward to demonstrate. First, consider the BN-VISTS trend equation re-arranged to be

$$
\boldsymbol{m}_{t}=\left(\boldsymbol{\mu}_{t}+A \boldsymbol{e}_{t}\right) /(1-L) .
$$

If $A=\gamma \boldsymbol{\delta}^{\prime}$, then $\boldsymbol{\mu}=\boldsymbol{\gamma} \boldsymbol{b}$ where $\boldsymbol{b}$ denotes a set of $k$ common drift terms. Therefore, 
equation (3.11) can be re-expressed as

$$
\boldsymbol{m}_{t}=\gamma\left(\boldsymbol{b}+\boldsymbol{\delta}^{\prime} \boldsymbol{e}_{t}\right) /(1-L)
$$

Recall that the BNSW common trend specification (equation (2.5)) defines the trend at time $t$ as

$$
\boldsymbol{\tau}_{t}=\left(\boldsymbol{b}+\boldsymbol{\delta}^{\prime} \varepsilon_{t}\right) /(1-L)
$$

Therefore equation (3.12) can be re-expressed as

$$
m_{t}=\gamma \tau_{t}
$$

\subsection{Estimation}

The likelihood derived in de Silva et al. (2007) can be employed in this context. The likelihood function is:

$$
L\left(\boldsymbol{\psi}, \boldsymbol{x}_{0}, \Sigma\right)=\left((2 \pi)^{T} \prod_{i=1}^{N} \sigma_{i}^{2}\right)^{-1 / 2} \exp \left(-\frac{1}{2} \sum_{t=1}^{T} \sum_{i=1}^{N} e_{i t}^{2} \sigma_{i}^{-2}\right)
$$

where $\boldsymbol{\psi}$ denotes the unknown coefficients, $\boldsymbol{x}_{0}$ denotes the state seed values and $\Sigma$ denotes a diagonal covariance matrix of the innovations. The non-zero (diagonal) elements of $\Sigma$ are denoted as $\sigma_{i}, i=1, \ldots, N$.

The initial state values, $\boldsymbol{x}_{0}$, are determined by running a regression on the first ten observations. The regression consists of a constant and a linear trend only. The constant is used for the initial trend value, $m_{0}$. The coefficient belonging to the linear time trend is reserved to be the starting value for $\mu$. The median of the residuals is employed for $c_{0}$ (and $c_{-1}$ when necessary).

The parameter matrices that feed into (3.13) to initiate the estimation procedure are diagonal. The diagonal values are set to $0.3,0.9,0.9$ and 0.25 for $A, \Phi_{1}, \Phi_{2}$ and $\Theta_{1}$ respectively.

In general, the likelihood is maximised subject to two conditions: one being invertibil- 
ity

$$
\bmod |\lambda(D)|<1
$$

where $D=F-A H$, and the other being stationarity

$$
\bmod \left|\lambda(F)_{N+1}\right|<1 \text {. }
$$

The invertibility condition denoted by equation (3.14) refers to the modulus of the largest eigenvalue of $D$. In relation to equation (3.15), it should be noted that the first $N$ eigenvalues are one by construction, the next largest eigenvalue (hence the subscript $N+1$ ), is therefore constrained to be of modulus less than one.

\subsection{Interpretability of the BN-VISTS specification}

A key advantage of the multivariate approach is that inter-relationships between components of different series can be gauged. The importance of this feature cannot be underestimated as it is naive to consider an economic variable in isolation. This is acknowledged by most researchers including Morley et al. (2003) who close their paper with a caveat stating that additional information may affect the estimates of the permanent and transitory component.

Correlation is a measure that is often used to gauge inter-series relationships. Although the specification adopted in this paper constrains the observations to be contemporaneously independent, the components are not. However, unlike the univariate case, the correlations between components of a given series are not necessarily perfect.

The degree of correlation is determined indirectly by the values of smoothing parameters. It is important here to distinguish between the two types of correlations often employed. The type being discussed is the correlation between the innovations not the correlation between estimates of the permanent and transitory components. The latter can be determined after the model is fitted, whereas the former is determined implicitly (Morley et al. 2003).

A special feature of the BN-VISTS formulation is that the effect of shocks can be traced out by using impulse response function analysis. Furthermore, the impulse response 
function of the transitory component also indicates the half-life of shocks.

The magnitude of the relative components is gauged by comparing the variance of each component. Different economic theories exist as to which component is more dominant. As already discussed, different specifications will yield different conclusions. Innovation specifications (i.e., ARIMA and innovation structural time series models, Morley et al. 2003) typically produce dominant permanent components. Most economists believe that the permanent component should be dominant in times of economic and political stability (Sinclair 2005).

In addition to the inter-series insights discussed so far, an interesting exercise is to compare official business cycle dates to the estimated permanent and transitory components. This type of comparison however, can be problematic and care should be taken when conducting this type of inference. In general there are two type of cycles, the classical business cycle and the growth cycle.

The classical business cycle is defined as the presence of peaks and troughs in the levels of the series. The growth cycle is defined similarly, however it is applied to the detrended levels. For a detailed discussion of this issue, refer to Pagan (1997).

In the following section, classical business cycle dates are considered. Although neither of the extracted components precisely match the classical business cycle definition, it is still an interesting comparison.

\section{Application of the Beveridge-Nelson Vector In- novations Structural Time Series models}

The data employed in this example are the quarterly real GDP for the UK, Australia and USA. Natural logarithms were taken before the models were fitted. These are displayed in Figure 1. The data covers the period from the third quarter of 1959 to the third quarter of 2007. This particular data set was used in Anderson et al. (2006). From the Australian perspective, the USA and the UK represent two very influential foreign economies.

The plot of the series indicate that real GDP is growing steadily over the 48 years. All three series are close in scale and therefore there is no need to standardise these series 


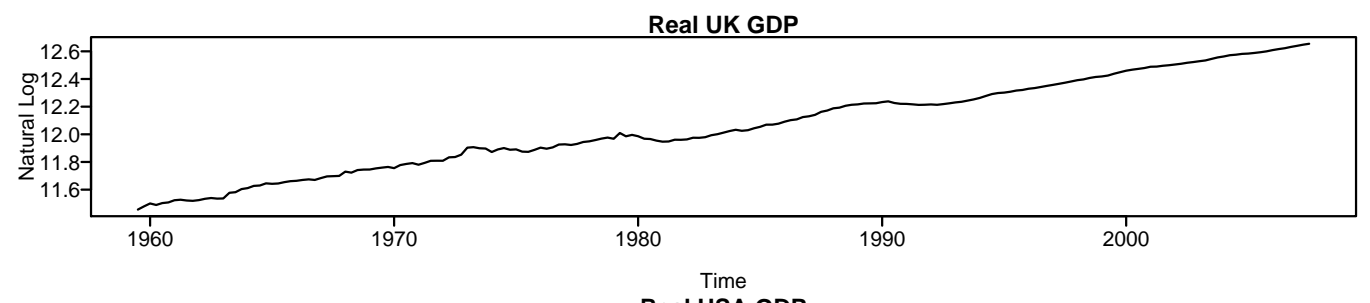

Real USA GDP

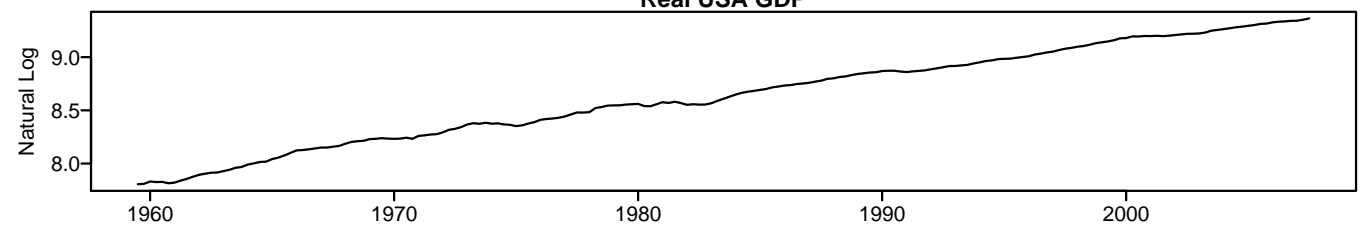

Time

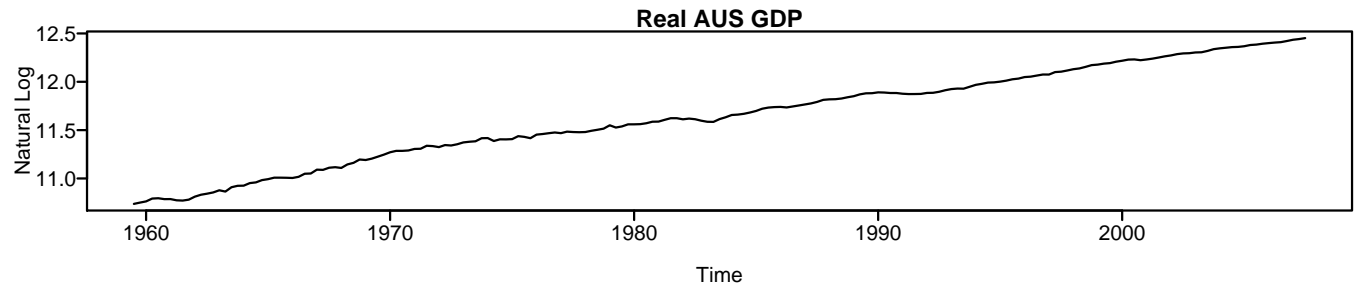

Figure 1: The Natural Logarithm of Real GDP of the UK, USA, \& Australia.

before the multivariate model is fitted.

The analysis is conducted in three stages. In the first stage a set of univariate models is fitted. In the second stage, an unrestricted multivariate version of the BN-VISTS framework is fitted. In the final stage, the smoothing matrix is constrained to be of reduced rank and therefore models two independent common features.

\subsection{Univariate model}

\subsubsection{First stage: univariate model}

As a first step the BN-UISTS models were fitted to the data. The results are slightly different from Anderson et al. (2006) as the sample size is larger and USA GDP not GNP is being employed.

The parameter estimates for each univariate model are displayed in Table 1. The columns of particular interest are $\alpha$ and $R^{2}$. These columns depict the direction and degree of the initial reaction in the long-run (permanent) component to a $1 \%$ change in GDP. Specifically, the $R^{2}$ values are calculated by regressing the first difference of the extracted permanent component against the first difference of the observations (this is in accordance with Anderson et al. 2006). Therefore, $R^{2}$ depicts the amount of variation 


\begin{tabular}{llrrrrrrrr} 
ARIMA order & Economy & $\phi_{1}$ & $\phi_{2}$ & $\theta_{1}$ & $\alpha$ & $\mu$ & $\sum_{i=1}^{T} e_{t}^{2}$ & $R^{2}$ & AIC \\
\hline$(0,1,1)$ & UK & & & & 0.968 & 0.007 & 4.305 & 0.755 & -10.032 \\
$(1,1,0)$ & UK & 0.037 & & & 0.955 & 0.009 & 4.302 & 0.965 & -10.033 \\
$(2,1,2)$ & UK & 0.381 & 0.328 & -0.455 & 1.620 & 0.007 & 4.001 & 0.641 & -10.732 \\
\hline$(0,1,1)$ & USA & & & & 1.173 & 0.008 & 6.779 & 0.951 & -9.578 \\
$(1,1,0)$ & USA & -0.249 & & & 1.331 & 0.008 & 6.646 & 0.686 & -9.598 \\
$(2,1,2)$ & USA & -0.030 & 0.427 & -0.361 & 1.594 & 0.008 & 6.403 & 0.895 & -9.604 \\
\hline$(0,1,1)$ & AUST & & & & 0.960 & 0.009 & 13.218 & 0.701 & -8.911 \\
$(1,1,0)$ & AUST & 0.037 & & & 0.965 & 0.009 & 13.223 & 0.995 & -8.910 \\
$(2,1,2)$ & AUST & 1.177 & -0.215 & 0.121 & 0.942 & 0.009 & 12.403 & 0.903 & -8.943 \\
\hline
\end{tabular}

Table 1: Univariate estimates.

captured by the trend component.

As previously stated, this model implicitly depicts the correlation between the latent components as being perfect. The direction of this correlation is ultimately determined by $\alpha$, which can be thought of as the long-run coefficient. If $\alpha>1$, then the correlation is negative. Conversely if $\alpha<1$, this implies that the correlation is positive. For the USA it appears that the permanent and the temporary components are perfectly negatively correlated. The opposite is true for Australia. The results for the UK however are inconclusive.

Interestingly, for each series considered the best BN-UISTS model is the BN$\operatorname{UISTS}(2,1,2)$. Therefore, a multivariate version of this model seems an appropriate extension.

\subsubsection{Second stage: multivariate model}

In this section the BN-VISTS $(2,1,2)$ is fitted. The form is slightly different from (3.10) as $A^{*}=I-A$. The reason for this becomes apparent in the third stage, where it is shown that there is no evidence for $A$ being of reduced rank, but there is for $A^{*}$. The resulting 
estimates are

$$
\begin{aligned}
& \hat{\Phi}_{1}=\operatorname{diag}\left[\begin{array}{lll}
0.756 & 0.195 & 0.684
\end{array}\right], \quad \hat{\Phi}_{2}=\operatorname{diag}\left[\begin{array}{lll}
0.485 & 0.155 & 0.035
\end{array}\right], \\
& \hat{\Theta_{1}}=\operatorname{diag}\left[\begin{array}{lll}
0.018 & 0.080 & 0.018
\end{array}\right], \quad \hat{\Sigma}=\operatorname{diag}\left[\begin{array}{lll}
8.918 & 6.724 & 12.303
\end{array}\right] \times 10^{3} \text {, } \\
& \hat{A}^{*}=\left[\begin{array}{rrr}
0.082 & -0.059 & -0.014 \\
-0.042 & -0.082 & -0.018 \\
-0.082 & 0.004 & 0.249
\end{array}\right], \quad I-\hat{A}^{*}=\left[\begin{array}{rrr}
0.918 & 0.059 & 0.014 \\
0.042 & 1.080 & 0.018 \\
0.082 & -0.004 & 0.751
\end{array}\right] \\
& \text { and } \hat{\boldsymbol{\mu}}=\left[\begin{array}{lll}
0.006 & 0.008 & 0.009
\end{array}\right] \text {. }
\end{aligned}
$$

\subsubsection{Third stage: multivariate common feature model}

The singular value decomposition decomposition is used to help identify common factors. It expresses any matrix as the product of three matrices, one of which is diagonal (cf. Schott 1997). The values in the diagonal matrix (which are sometimes referred to as singular values) are typically arranged in descending order and can be interpreted in a similar way to eigenvalues; a value of zero indicates the matrix is of reduced rank.

The singular values of $A^{*}$ are $0.272,0.100$ and 0.069 . Although none of these values are zero, the smallest value, 0.069 , is very close to zero. Conversely, the three singular values relating to $A$ are $1.095,0.900$ and 0.726 . Therefore it seems appropriate to constrain $A^{*}$ to have a rank of two and hence

$$
A^{*}=\boldsymbol{\alpha} \boldsymbol{\beta}^{\prime}
$$

Restricting the rank of $A^{*}$ means it has the structure depicted by equation (4.1). As a result the likelihood must be maximised conditional on $\boldsymbol{\alpha}$ or $\boldsymbol{\beta}$, as they cannot be uniquely determined. We chose to specify $\boldsymbol{\beta}$ according to economic theory prior to fitting the new model.

Specifically, the three economic variables considered in this example all measure the level of economic activity. In other words, GDP can be loosely considered as an economic barometer. As these economies are all developed, and have strong trade and political links, it is reasonable to expect them to move together over time. This is confirmed visually by Figure 1. In other words, $\boldsymbol{\beta}$ should specify error correction mechanisms between the 
series, thereby constraining the real GDP values to move together over time:

$$
\boldsymbol{\beta}=\left[\begin{array}{rr}
1 & 0 \\
-1 & 1 \\
0 & -1
\end{array}\right]
$$

This restriction shows that two common trends are present. The trends are specified to be error correcting mechanisms constraining two of the three series to move together over time. Enforcing this restriction yields the following parameter estimates:

$$
\begin{aligned}
& \hat{\Phi}_{1}=\operatorname{diag}\left[\begin{array}{lll}
0.014 & -0.442 & 0.138
\end{array}\right], \quad \hat{\Phi}_{2}=\operatorname{diag}\left[\begin{array}{lll}
0.304 & 0.349 & -0.429
\end{array}\right], \\
& \hat{\Theta_{1}}=\operatorname{diag}\left[\begin{array}{lll}
-0.062 & 0.006 & 3.636
\end{array}\right], \hat{\Sigma}=\operatorname{diag}\left[\begin{array}{lll}
8.824 & 6.496 & 11.528
\end{array}\right] \times 10^{3} \text {, } \\
& \hat{\boldsymbol{\alpha}}=\left[\begin{array}{rr}
0.094 & -0.009 \\
0.016 & -0.236 \\
-0.178 & -0.186
\end{array}\right], \text { and } \hat{\boldsymbol{\mu}}=\left[\begin{array}{lll}
0.006 & 0.008 & 0.009
\end{array}\right] \text {, }
\end{aligned}
$$

where the estimates in $\hat{\boldsymbol{\alpha}}$ represent how quickly the temporary component adjusts to country specific shocks. Essentially the values in $\hat{\boldsymbol{\alpha}}$ can be interpreted as speed of adjustment parameters (Enders 1995, page 394) Before the model can be interpreted, however, the appropriateness of the restriction (4.2) is evaluated.

The restriction can be evaluated in two ways. Either conducting a likelihood test or using an information criterion. The AIC for the unrestricted and restricted model is -27.718 and -27.859 respectively. Therefore we can conclude that there is evidence that the restriction is valid.

Table 2 presents the implied correlations between the latent innovations where the $m$ and $c$ subscripts correspond to the trend and transitory components. The lower triangle of the table indicates the direction of the linear association. The correlations are calculated 
using the conditional variance of the state components, that is:

$$
\operatorname{var}\left(\boldsymbol{x}_{t} \mid I_{t-1}\right)=\left[\begin{array}{c}
I-A^{*} \\
\cdots \\
A^{*}
\end{array}\right] \Sigma\left[I-A^{*} \quad \vdots \quad A^{*}\right]
$$

\begin{tabular}{lrrrrrr} 
& $\mathrm{UK}_{m}$ & $\mathrm{USA}_{m}$ & $\mathrm{AUS}_{m}$ & $\mathrm{UK}_{c}$ & $\mathrm{USA}_{c}$ & $\mathrm{AUS}_{c}$ \\
\hline $\mathrm{UK}_{m}$ & & 0.082 & 0.176 & 0.655 & -0.023 & -0.650 \\
$\mathrm{USA}_{m}$ & + & & -0.235 & -0.693 & -0.797 & -0.201 \\
$\mathrm{AUS}_{m}$ & + & - & & 0.210 & 0.770 & 0.633 \\
$\mathrm{UK}_{c}$ & + & - & + & & 0.524 & -0.387 \\
$\mathrm{USA}_{c}$ & - & - & + & + & & 0.583 \\
$\mathrm{AUS}_{c}$ & - & - & + & - & + &
\end{tabular}

Table 2: Correlation between latent innovations. The $m$ and $c$ subscripts denote the trend and transitory components respectively.

According to Table 2, the correlation between the permanent and temporary components of USA GDP is negative. If real shocks play a dominant role, this suggests short term (temporary) fluctuations largely reflect adjustments towards a shifting trend (Stock \& Watson 1988b). This finding is consistent with Proietti (2002), Morley et al. (2003) and Sinclair (2005). Interestingly, a negative correlation is also observed between the USA permanent component and the temporary components of the UK and Australia. This suggests adjustments in the UK and Australian temporary components may be reflecting real shocks in the USA economy. The estimated correlations between the permanent component of UK GDP and the components of USA GDP are very small, indicating that UK GDP is relatively insensitive to USA GDP.

Caution must be exercised when interpreting these results as the components are interdependent making it difficult to isolate the overall effect of one variable upon another. To address this problem, we perform impulse response function analysis which traces the effect of a shock on a given variable.

The impulse response functions of the transitory component are presented in Figure 2. For the sake of brevity, only the responses in the temporary component to a negative $1 \%$ shock in USA GDP are shown.

The insensitivity of the UK economy with respect to the US economy is once again 

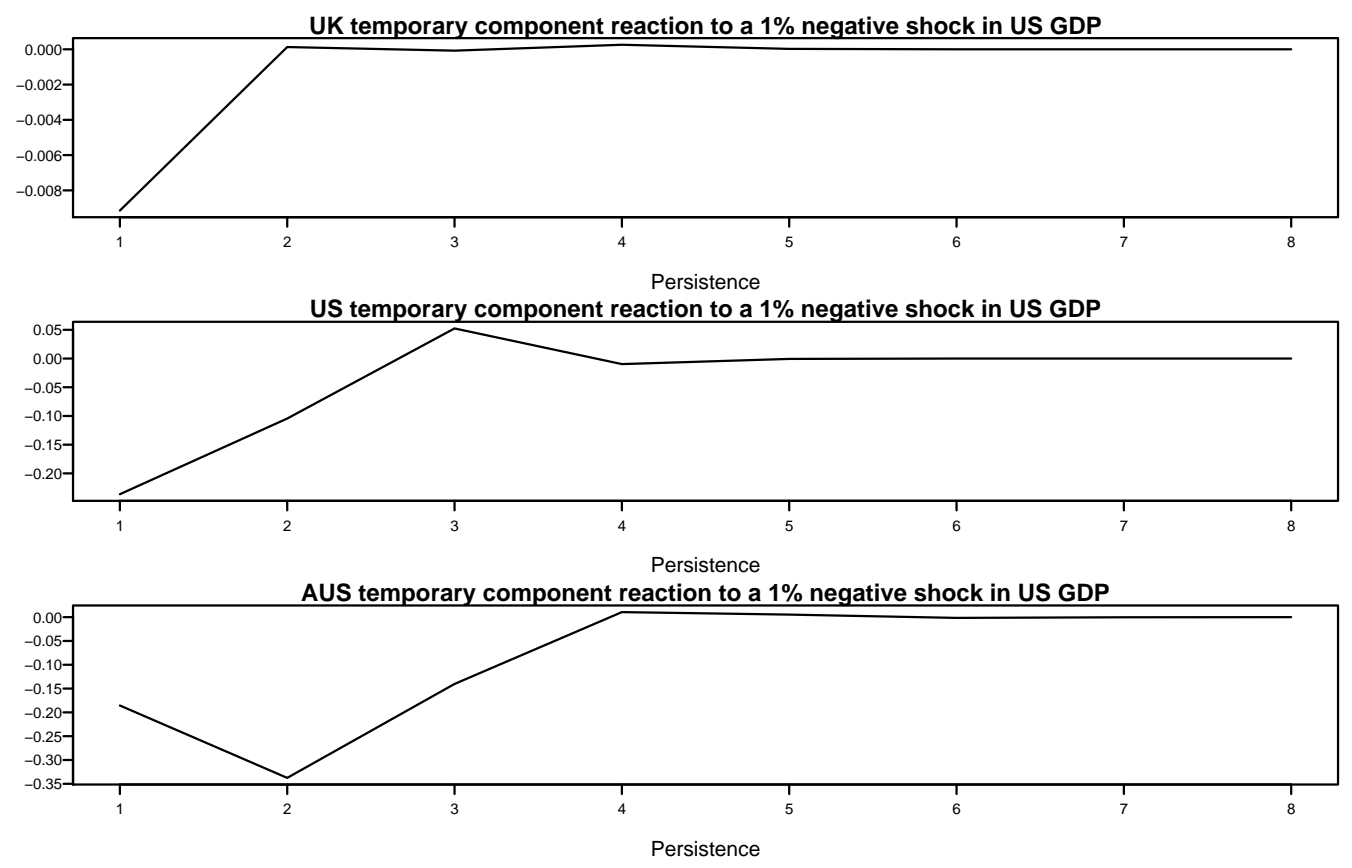

Figure 2: Impulse Response Function of temporary components.

borne out. In particular the shock to UK GDP is short lived and small in magnitude. This makes intuitive sense as the UK economy is more dependent on European economies than the USA economy.

In contrast the Australian economy which is often categorised as a small open economy is very sensitive to the USA. The USA over the period of this data has been considered to be Australia's most important economic partner. Therefore, the large drop and slow return is expected. A particular interesting feature of the impulse response function is the full extent of the shock is only reached in the second period. This suggests that a shock in USA GDP will have a significant and prolonged effect on Australian GDP.

We conclude this section by comparing the estimated components to cycle dates. In Figure 3 the estimated latent components are displayed. The grey shading indicates periods of recession (peak to trough episodes) as identified by the Economic Cycle Research Institute (2007). Although the peak to trough episodes differ for each economy, in general the recessions coincide with a flattening out or a decrease in the permanent component.

There appears to be little association between the estimated transitory component and the recessionary periods. However, it is apparent that the volatility in the temporary 

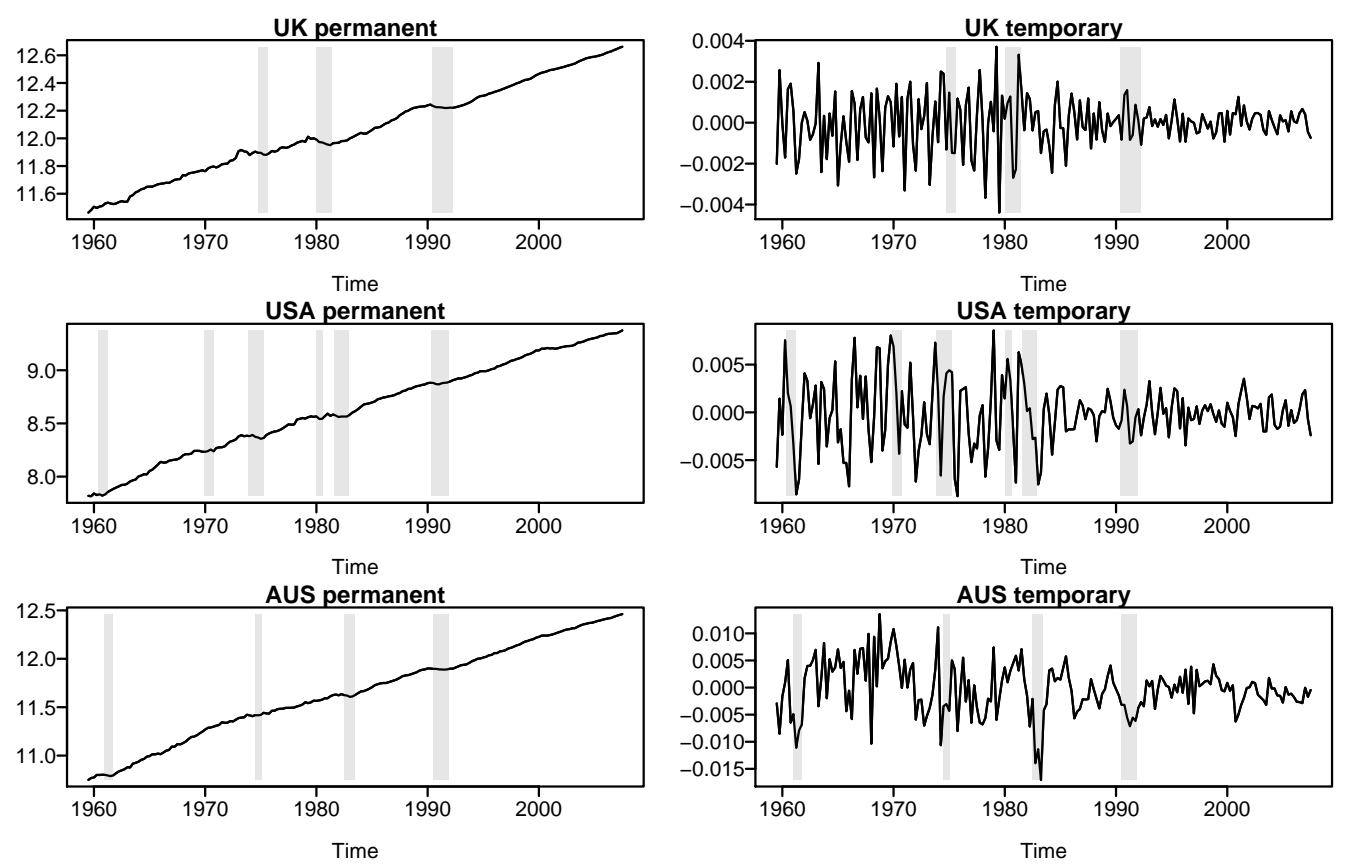

Figure 3: Comparision of latent components with ECRI recession dates.

component has decreased post 1985. Interestingly, this period is also characterised by fewer recessionary periods (Economic Cycle Research Institute 2007).

In general, it may be argued that the estimated latent components are consistent with the state of the economy as indicated by ECRI. This is an important observation as it helps verify the proposed decomposition.

\section{Conclusion}

This paper has illustrated a multivariate $\mathrm{BN}$ decomposition that is simple and flexible. This new approach is an extension of the newly introduced vector innovations structural time series framework (de Silva et al. 2007). It has been shown that the inference from this new approach is consistent with economic rationale.

Specifically, this analysis has shown how to exploit inter-series relationships. Two key features have been illustrated: first the capability to model inter-series dependencies, and second, the ability to capture common features between series. 


\section{References}

Anderson, H., Low, C. \& Snyder, R. (2004), Single source of error state space approach to the beveridge nelson decomposition, Technical report, Department of Econometrics and Business Statistics.

Anderson, H., Low, C. \& Snyder, R. (2006), 'Single source of error state space approach to Beveridge-Nelson decomposition', Economics Letters 91, 104-109.

Athanasopoulos, G. (2005), Essays on alternative methods of identification and estimation of vector autoregressive moving average models, $\mathrm{PhD}$ thesis, Department of Econometrics and Business Statistics, Monash University, Australia.

Barillas, F. \& Schleicher, C. (2003), Common trends and common cycles in Canadian sectoral output, Working paper 2003-44, Bank of Canada.

Beveridge, S. \& Nelson, C. (1981), 'A new approach to decomposition of economic times series into permanent and transitory components with particular attention to the measurement of the business cycle', Journal of Monetary Economics 7, 151-174.

de Silva, A., Hyndman, R. \& Snyder, R. (2007), The Vector Innovation Structural Time Series Framework: A Simple Approach to Multivariate Forecasting, Working Paper 03/2007, Department of Econometrics and Business Statistics, Monash University.

Dufour, J.-M. \& Pelletier, D. (2005), Practical methods for modelling weak VARMA processes: identification, estimation and specification with a macroeconomic application, Discussion paper, CIRANO and CIREQ, Universite de Montreal.

Economic Cycle Research Institute (2007). http://www.businesscycle.com/.

Enders, W. (1995), Applied econometric time series, 1st edn, John Wiley \& Sons.

Harvey, A. \& Jaeger, A. (1993), 'Detrending, stylised facts and the business cycle', Journal of Applied Econometrics 8, 231-247.

Hyndman, R. J., Koehler, A. B., Ord, J. K. \& Snyder, R. D. (2008), Forecasting with exponential smoothing: the state space approach, Springer. 
Hyndman, R. J., Koehler, A. B., Snyder, R. D. \& Grose, S. (2002), 'A state space framework for automatic forecasting using exponential smoothing methods', International Journal of Forecasting 18, 439-454.

Johansen, S. (1995), Likelihood-Based Inference in Cointegrated Vector Autoregressive Models, Oxford University Press.

King, R., Plosser, C., Stock, J. \& Watson, M. (1987), Stochastic trends and economic fluctuations, NBER Working Paper 2229, NBER.

Morley, J. C. (2002), 'A state-space approach to calculating the Beveridge-Nelson decomposition', Economics Letters 75, 123-127.

Morley, J. C., Nelson, C. R. \& Zivot, E. (2003), 'Why are the Beveridge-Nelson and unobserved-components decompositions of GDP so different?', The Review of Economics and Statistics 75(2), 235-243.

Pagan, A. (1997), 'Towards an understanding of some business cycle facts', The Australian Economic Review 30, 1-15.

Proietti, T. (2002), Some reflections on trend-cycle decompositions with correlated components, Working Paper, Department of Economics, European University Institute.

Schott, J. (1997), Matrix Analysis for Statistics, John Wiley \& Sons.

Sinclair, T. (2005), Permanent and transitory movements in output and unemployment: Okun's law persists, Working Paper, Department of Economics, Washington University.

Stock, J. \& Watson, M. (1988a), 'Testing for common trends', Journal of the American Statistical Association 83(404), 1097-1107.

Stock, J. \& Watson, M. (1988b), 'Variable trends in economic time series', Journal of Economic Perspectives 2, 147-174.

Tiao, G. \& Tsay, R. (1989), 'Model specification in multivariate time series', Journal of the Royal Statistical Society, Series B 51, 157-213. 
Vahid, F. \& Engle, R. (1993), 'Common trends and common cycles', Journal of Applied Econometrics 8(4), 341-360.

Vahid, F. \& Engle, R. (1997), 'Codependent cycles', Journal of Econometrics 80, 199-221.

Watson, M. (1994), Vector Autoregressions and Cointegration, Vol. IV of Handbook of Econometrics, Elsevier, chapter 47, pp. 2843-2915. 